\title{
Emergência em saúde pública por inundações: a atuação do Ministério da Saúde em ocorrências no Brasil de 2004 a 2017
}

\author{
Emergency in public health caused by floods: the Ministry of Health \\ actions in occurrences in Brazil, from 2004 to 2017
}

Eliane Lima e Silva1, Rodrigo Matias de Sousa Resende $\mathbf{2}$, Rodrigo Lins Frutuoso², Amarílis Bahia Bezerra², Barbara Bresani Salvi², Daniela Buosi Rohlfs²

DOI: 10.1590/0103-11042020E212

RESUMO Este artigo teve por objetivos contextualizar os impactos das inundações na saúde e analisar relatórios do Centro de Operações de Emergência em Saúde, mobilizados pelo Ministério da Saúde (MS), para monitoramento federal desses eventos no Brasil, de 2004 a 2017. Para isso, foi realizado levantamento bibliográfico e documental, incluindo relatórios do MS sobre inundações, e feita análise de dados do Sistema Integrado de Informações sobre Desastres, da Defesa Civil, no referido período. Verificou-se que as inundações atingiram todas as regiões brasileiras, com eventos críticos em 2004, 2009, 2010 e 2011. O MS atuou em nove ocorrências, e essa experiência subsidiou o estabelecimento da estratégia de preparação e resposta, incluindo Comitês de Saúde em Desastres, documentos e normativas para orientar a atividade do Sistema Único de Saúde (SUS) na atuação em desastres hidrológicos. Inundações de grande magnitude exigem resposta rápida, e isso prescinde de preparação prévia. O MS avançou nas articulações intersetoriais e interinstitucionais, no entanto, dotar o SUS municipal da capacidade necessária para atuação oportuna apresenta-se ainda como um desafio a ser superado.

PALAVRAS-CHAVE Desastres naturais. Inundações. Saúde pública. Gestão de riscos. Saúde ambiental.

\begin{abstract}
The objective of this article is to contextualize the impacts of floods on health and to analyze reports from the Emergency Health Operations Center, mobilized by the Ministry of Health (MS), for the federal monitoring of these events in Brazil, between 2004 and 2017. For such, a bibliographical and documentary survey was carried out, including MS reports on floods and data analysis of the Integrated Disaster Information System, from the Civil Defense, from 2004 to 2017. It was verified that floods reached all Brazilian regions, with critical events in 2004,2009, 2010, and 2011. The MS worked in nine occurrences and this experience subsidized the establishment of the preparedness and response strategy, including Health in Disaster Committees, documents, and regulations to guide the SUS's (Unified Health System) action in hydrological disasters. Floods of great magnitude require rapid response and this does not require prior preparation. The MS has advanced in inter-sectorial and interinstitutional articulations, however, providing the municipal SUS with the necessary capacity for timely action is still a challenge to be overcome.
\end{abstract}

(UnB), Departamento de Geografia (GEA), Laboratório de Geografia, Ambiente e Saúde (Lagas)

- Brasília, DF, Brasil.

2 Ministério da Saúde (MS), Secretaria de Vigilância em Saúde (SVS) - Brasília (DF), Brasil. rodrigo.msr@hotmail.com 


\section{Introdução}

A redução do risco de desastres está entre as funções essenciais de saúde pública, ${ }^{\mathbf{1} 2}$ uma vez que estes provocam impactos diretos e indiretos sobre a saúde das pessoas e sobre os serviços de saúde ${ }^{\mathbf{3}, 4}$, exigindo a organização do setor saúde para atuar de forma oportuna nessas situações ${ }^{1,5}$.

As inundações são classificadas como desastres naturais de cunho hidrológico, conforme estabelece a Classificação e Codificação Brasileira de Desastres, e são compreendidas como o transbordamento de água da calha normal de cursos d'água ou sua acumulação por problemas na drenagem em áreas normalmente não submersas, podendo se apresentar de forma gradual ou súbita ${ }^{6}$, característica esta que define os danos à saúde ${ }^{1,3,7}$. Elas estão entre os desastres mais recorrentes no mundo, e a dimensão dos seus impactos dependem das condições de vulnerabilidades presentes no território atingido ${ }^{2,7}$.

No Brasil, as inundações são recorrentes em todas as regiões, atingem mais de $50 \%$ dos municípios e se distribuem ao longo de todo ano ${ }^{\mathbf{1 , 2}, \mathbf{8}, \mathbf{9}}$. Entre os impactos sobre a saúde, estão: os óbitos, os traumas e lesões, as doenças por contato com a água contaminada, os transtornos psicológicos ${ }^{1-4,7}$; além do potencial comprometimento dos serviços de saúde, seja por danos diretos às unidades de atendimento, seja pelo acesso a elas, 4,5,-11. Quanto mais vulneráveis forem as comunidades das áreas atingidas por inundações, maiores são os impactos sobre a saúde $\mathrm{i}^{\mathbf{1}, \mathbf{9}, \mathbf{1 2}, \mathbf{1 3}}$.

Nesse cenário de inundações recorrentes distribuídas por todo o País e com potencial de danos, para que o setor saúde atue de forma oportuna, tanto com ações preventivas e de redução do risco quanto de resposta, é necessário o estabelecimento de estratégias de atuação que envolvam as três esferas de gestão do Sistema Único de Saúde (SUS). Nesse intuito, o Ministério da Saúde (MS) definiu uma estratégia de preparação para resposta à Emergência em Saúde Pública (ESP), incluindo aquelas decorrentes de desastres, em que estabeleceu diretrizes para a organização do SUS ${ }^{\mathbf{1 4}}$.

Para a articulação e harmonização das ações de resposta em eventos de grande magnitude, adota-se o acionamento do Centro de Operações de Emergência em Saúde (COE). Assim, os objetivos deste artigo são: contextualizar os impactos das inundações na saúde e analisar relatórios do COE, mobilizados pelo MS para monitoramento federal desses eventos no Brasil, de 2004 a 2017.

\section{Material e métodos}

$\mathrm{O}$ artigo apresenta um estudo descritivo que foi estruturado e redigido com base em pesquisa bibliográfica e documental, com revisão da literatura científica sobre o tema e a análise dos relatórios elaborados pelo COE, mobilizados no âmbito do MS, para o monitoramento da resposta do SUS à ESP por inundações ocorridas no período de 2004 a 2017.

Para a caracterização espaço-temporal da ocorrência de inundações no Brasil, foram analisados os registros de eventos hidrológicos do Sistema Integrado de Informações sobre Desastres (S2ID) da Secretaria Nacional de Proteção e Defesa Civil (Sedec), ocorridos no período de janeiro de 1998 a julho de 2017.

Para a compreensão do comportamento das inundações e seus potenciais impactos sobre a saúde, foram analisados publicações científicas e documentos governamentais norteadores da gestão do risco de desastres hidrológicos, envolvendo o contexto internacional e o nacional.

\section{Resultados e discussão}

A variabilidade espaço-temporal das inundações condiciona ou determina processos ecológicos e influencia o comportamento antrópico. Em um contexto macroeconômico, podem comprometer a infraestrutura e a economia; enquanto em um nível microeconômico, afetam o acesso à educação e à saúde, 
culminando em impactos socioeconômicos ${ }^{15}$. Compreender esses eventos propicia parte do subsídio necessário para a adoção de medidas que reduzam o risco de exposição das pessoas, já que as inundações podem resultar em emergências e desastres quando as áreas inundáveis são habitadas $\mathbf{1 , 2}^{\mathbf{2}}$.

\section{Cenário internacional e nacional de eventos hidrológicos}

Os desastres provocados por eventos hidrológicos se apresentam como as ocorrências mais comuns e podem gerar impactos de grandes dimensões com potencial para perdurar ao longo do tempo ${ }^{2,15}$. Estudo das Nações Unidas apontou que, em 20 anos, considerando o contexto internacional, entre 1998 e
2017, os desastres relacionados com o clima representaram $91 \%$ dos registros, sendo $43,4 \%$ inundações e $28,8 \%$ tempestades; observou-se também um aumento de $151 \%$ nas perdas econômicas por desastres climáticos, em relação ao período de 1978 a 1997, e os países atingidos relataram quase US\$ 3 bilhões de prejuízos16.

No Brasil, no mesmo período estudado pela Organização das Nações Unidas (ONU) (1998 a 2017), foram registrados 13.479 eventos de origem natural, e os hidrológicos figuram em segundo lugar, com 29,8\% das ocorrências, superado apenas pelos climatológicos. Ao longo do período, houve picos de ocorrências de inundações com destaque para 2004 e para o quadriênio 2008 a 2011, em que 2009 se destaca como o pior registro histórico, com 1.695 registros em todo o País, conforme gráfico 1.

Gráfico 1. Quantitativo de registros de eventos hidrológicos no Brasil, no período de 1998 a 2017

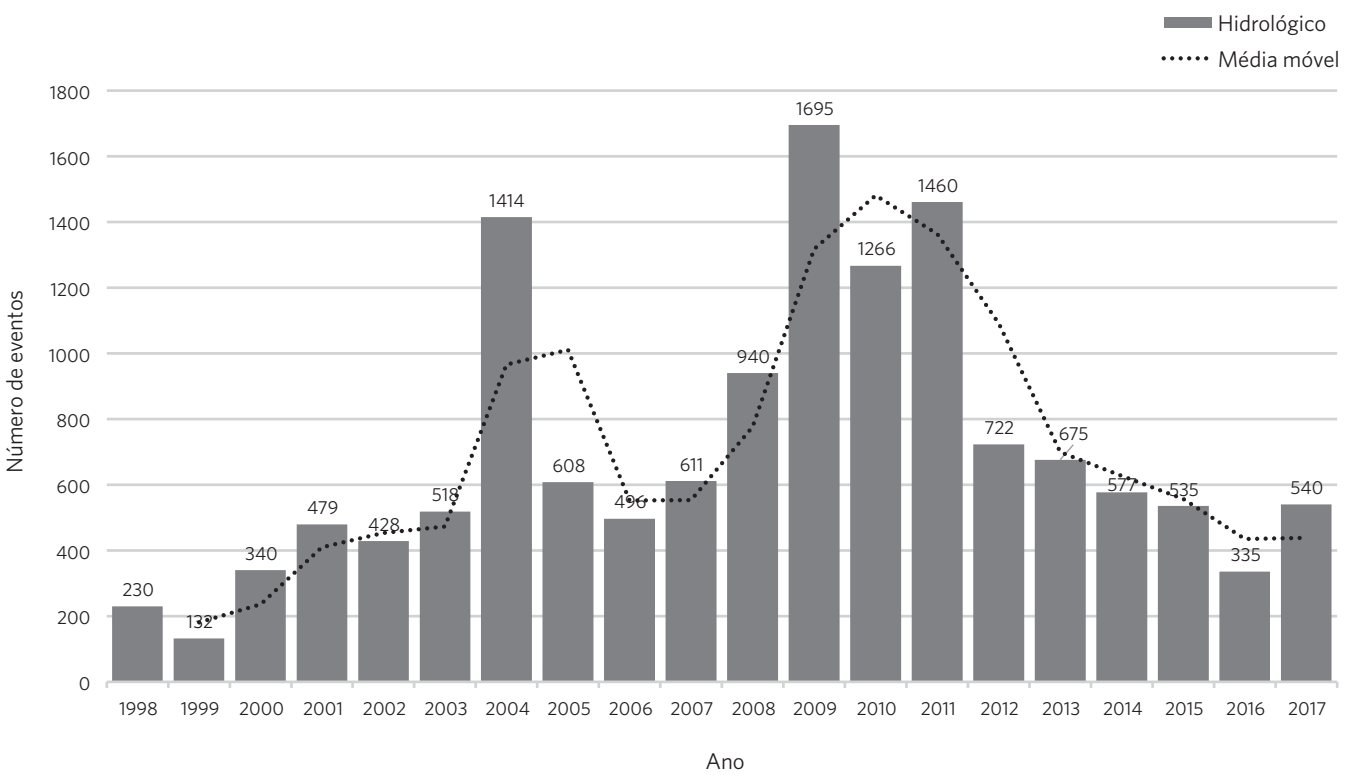

Fonte: Elaboração própria.

A distribuição espacial dessas ocorrências (figura 1) atingiu todo o Brasil, principalmente as regiões Norte, Sudeste e Sul, o que aponta para a necessidade de que os serviços públicos se organizassem para ampliar a sua capacidade de atuação e a adoção de medidas oportunas para se prepararem e responderem às emergências e desastres de forma efetiva. 
Figura 1. Distribuição espacial dos registros de eventos hidrológicos no Brasil, no período de 1998 a 2017

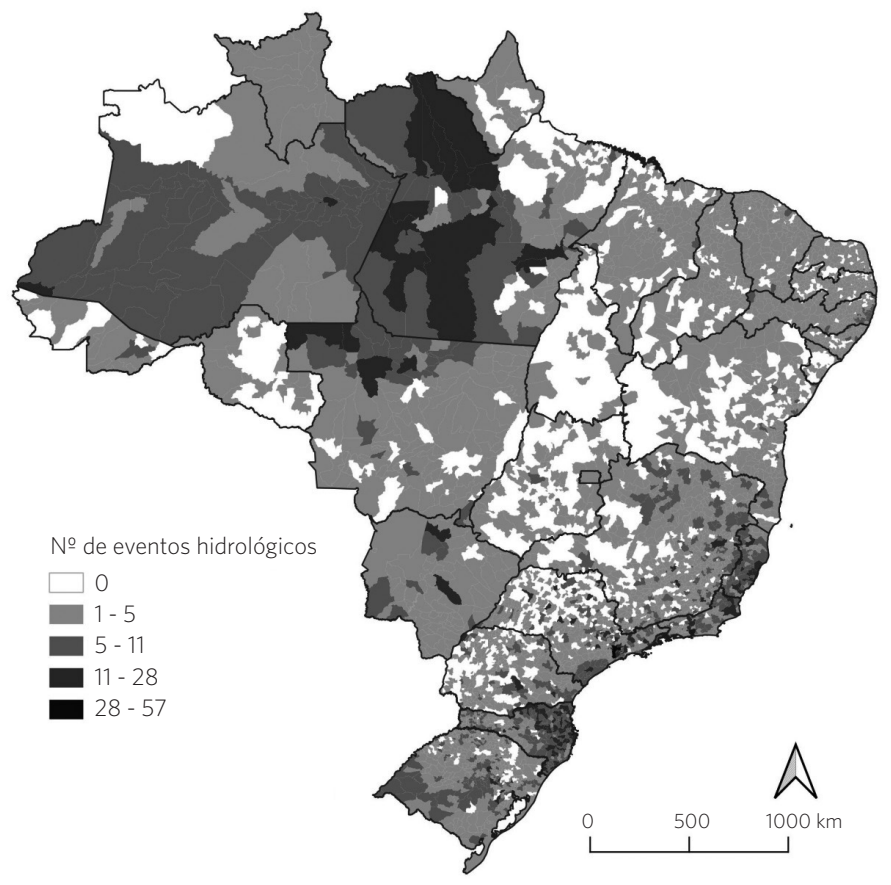

Fonte: Elaboração própria a partir de dados do Sistema Integrado de Informações sobre Desastres (S2ID).

\section{Impactos sobre a saúde}

Os impactos das inundações sobre a saúde podem se apresentar de forma direta ou indireta, de curto, médio e longo prazo; e atingem o indivíduo e a comunidade, tornando-se um problema de saúde pública ${ }^{3,4}$. A forma súbita ou gradual das ocorrências também influencia no comportamento e na dimensão dos danos, em que têm destaque os óbitos por afogamento ou trauma, doenças transmissíveis (hídrica e alimentar e vetores), acidentes com animais peçonhentos, choques elétricos, transtornos psicossociais, entre outros ${ }^{1,7}$. Os grupos mais vulneráveis são as crianças, os idosos e as pessoas com deficiência ou com limitação de locomoção e as gestantes $\mathbf{1 , 5 , 7 , 9 , 1 0 , 1 5}$.

A dimensão desses impactos está diretamente relacionada com as condições de vulnerabilidades presentes na área atingida ${ }^{15,17}$ bem como com a capacidade de atuação oportuna dos atores envolvidos na resposta à emergênciaa1,18. No âmbito da saúde, a discussão sobre a necessidade de ampliar as capacidades para uma atuação oportuna em emergências e desastres não é recente, sendo ampliada a partir da divulgação dos relatórios do Painel Intergovernamental sobre Mudanças Climáticas, quando estes apontaram que extremos climáticos serão cada vez mais recorrentes ${ }^{1,19}$.

A busca por evidências epidemiológicas de que as inundações provocam impactos à saúde tem apresentado alguns achados, em que os mais evidentes são a ocorrência de óbitos (afogamento, choque elétrico ou trauma); lesões (contusões, lacerações ou fraturas); doenças transmissíveis (fecal-oral e vetores) 7,10,20-22 . Entre os óbitos, em áreas de alta renda, eles estão associados ao afogamento em automóveis; e quando registrados em residências, a maioria é de idosos ${ }^{7}$. 
O contato com a água contaminada pode provocar diversas doenças, com destaque para as de transmissão fecal-oral (diarreias, rotavírus, hepatites, gastroenterites), vetores (dengue, hantavirose e leptospirose). A saúde mental também pode ser influenciada pelas inundações, sendo identificados eventos envolvendo síndrome do estresse pós-traumático, ansiedade, irritabilidade, agressividade, insônia, depressão e suicídio ${ }^{4,7,10,20}$. Contudo, estabelecer a relação entre inundações e ocorrência de doenças é complexo uma vez que os dados são subestimados; e os registros, escassos ${ }^{7}$. Os riscos imediatos de trauma e morte são geralmente claros, em detrimento dos impactos de longo prazo, em especial, sobre saúde mental7,10.

\section{Ampliação da capacidade de atuação e organização da resposta a emergências e desastres}

Atuação oportuna exige preparação prévia ${ }^{18,19}$. A formulação de políticas públicas de redução do risco à saúde é limitada pela escassez de evidências sobre fatores de risco epidemiológicos e intervenções de saúde pública; pela não quantificação dos riscos de doenças infecciosas e transmitidas por vetores após as inundações; pela não identificação dos impactos de médio e longo prazo, pouco estudados; pelos sistemas de alerta ineficientes e pela não inserção dos custos sobre a saúde nas análises de impacto ${ }^{\mathbf{7}, \mathbf{1 9}, 21}$.

Nesse contexto, o desenvolvimento e a implementação de políticas públicas relacionadas com a gestão do risco de desastres são um desafio enfrentado por diversos países ${ }^{18,19}$. Estimar os impactos potenciais dos desastres naturais é de suma importância para pesquisadores e acadêmicos, mas principalmente para formuladores de políticas e governos ${ }^{15}$. $\mathrm{O}$ fortalecimento da capacidade para resposta a emergências e desastres é apontado como necessário desde a década de 1970, e uma das iniciativas precursoras para isso foi a criação do Programa de Preparativos para Situações de
Emergência e Resposta a Desastres em Saúde na Organização Pan-Americana da Saúde (Opas) ${ }^{19}$, que ressalta que as especificidades locais devem ser consideradas para o fortalecimento da atuação do setor saúde nessa temática, abrangendo como subsídio para a tomada de decisão o histórico espaço-temporal da ocorrência dos eventos, bem como as notificações de doenças e agravos à saúde.

No Brasil, a partir da publicação da Política Nacional de Proteção e Defesa Civil, instituída pela Lei ${ }^{\circ} 12.608 / 2012$, alguns avanços institucionais foram alcançados, destacando-se a abordagem sistêmica das ações de prevenção, mitigação, preparação, resposta e recuperação ante os desastres, naturais e/ou tecnológicos ${ }^{23}$.

Dentre as diretrizes internacionais, o Marco de Sendai para a redução do risco de desastres 2015-2030 aponta como um desafio a ampliação na abordagem de atuação dos órgãos governamentais, centrada nas pessoas para prevenir os riscos de desastres, considerando estratégias multissetoriais, inclusivas e acessíveis ${ }^{\mathbf{1 8}}$.

Dessa forma, a atuação do SUS em desastres hidrológicos deve estar baseada na prevenção de riscos, incluindo uma abordagem integral sobre o cuidado, planejamento das atividades propostas, avaliação dos impactos gerados, direta ou indiretamente, e, ainda, a sensibilidade dos serviços de saúde para mudanças de comportamento no perfil epidemiológico, durante e após o evento $5,11,14$.

A perspectiva de risco deve, ainda, ser avaliada e estar em consonância com o desenvolvimento das políticas públicas de saúde no território, de forma que a atuação do SUS seja efetiva e oportuna, considerando a realidade local e a demanda pelos serviços de saúde ${ }^{2,11}$.

\section{Atuacão do Ministério da Saúde em inundações}

A preparação para a resposta à ESP por inundações tem sido amplamente discutida no MS, que estabeleceu uma estratégia de gestão de risco para emergência e desastres que envolvem ações a serem adotadas de forma contínua 
e permanente pelas autoridades de saúde pública para a redução do risco, o manejo da emergência e a recuperação dos seus efeitos, conforme preconiza a Estratégia Internacional de Redução de Desastres, o Regulamento Sanitário Internacional e a Política Nacional de Proteção e Defesa Civil5,11.

Em 2012, o governo federal lançou o Plano Nacional de Gestão de Riscos e Resposta a Desastres Naturais, com ações de prevenção (obras estruturantes), mapeamento de áreas de risco, monitoramento-alerta e de resposta e reconstrução para deslizamentos, enchentes e secas, que envolveu diversos atores, entre eles, o MS, o que subsidiou ainda mais o estabelecimento da estratégia de preparação e resposta do MS para ESP14,24.

Observando a regulamentação legal das ações em situações de desastres, identifica-se que a atuação do setor saúde no atendimento à emergência remonta à própria história da saúde pública ${ }^{3}$. A necessidade de dotar o SUS da capacidade necessária para atuar de forma oportuna em inundações foi evidenciada em 2004 quando mais de mil municípios foram severamente atingidos por inundações no Brasil e o MS mobilizou uma sala de situação para gerenciar o atendimento às secretarias de saúde, uma vez que tiveram sua capacidade de resposta superada, necessitando de apoio adicional do governo federal25. A mesma iniciativa se repetiu para responder às inundações e deslizamentos que atingiram Santa Catarina (2008), para inundações em Minas Gerais e Espírito Santo (2010), nas enxurradas e deslizamentos da Região Serrana do estado do Rio de Janeiro (2011), em inundações no Acre e no Amazonas (2012), nas enxurradas em Lajedinho, na Bahia (2013) e nas inundações que atingiram o Acre e Rondônia em 2014, conforme disposto no quadro 1, resultado da análise dos relatórios de acompanhamento desses eventos.

Quadro 1. Síntese dos relatórios dos Centros de Operações de Emergência em Saúde para gestão de ações de reposta a inundações, 2004 a 2017

\begin{tabular}{|c|c|c|c|}
\hline Ano & Evento & Principais Impactos & Principais Medidas \\
\hline 2004 & $\begin{array}{l}\text { Inundações no } \\
\text { Brasil }\end{array}$ & $\begin{array}{l}\text { Chuvas no verão de } 2004 \text { provocaram } \\
\text { inundações em todas as regiões do Brasil, } \\
\text { com mais intensidade nas regiões Sudeste } \\
\text { e Nordeste, atingindo } 1.224 \text { municípios. } \\
\text { No período de } 28 \text { de janeiro a } 22 \text { de março } \\
\text { de } 2004 \text {, foram registrados } 211 \text { óbitos, } \\
1.387 \text { feridos e } 378.557 \text { pessoas afetadas, } \\
\text { entre desabrigados e desalojados. Foram } \\
\text { atingidas (danificadas/destruídas) } 113 \text { mil } \\
\text { residências, } 1.046 \text { pontes e } 1.838 \text { escolas } \\
\text { públicas. Houve danos em unidade de } \\
\text { saúde. }\end{array}$ & $\begin{array}{l}\text { Instituído gabinete de crise na Casa Civil da Presidência da República com } \\
\text { mobilização de diversos setores no intuito de discutir, aprovar e planejar as } \\
\text { ações federais, recomendar prioridades, consolidar as informações recebi- } \\
\text { das, além de acompanhar as ações setoriais, com prioridade para o acesso à } \\
\text { alimentação e água potável, suprimento de medicamentos básicos, garantia } \\
\text { para o não isolamento de comunidades e que escolas públicas mantivessem } \\
\text { condições de funcionamento. } \\
\text { Para o monitoramento, o MS instituiu Grupo de Trabalho, Portaria GM no } \\
\text { 284/2004, intersetorial para a intensificação das ações de Vigilância em } \\
\text { Saúde (saúde ambiental, doenças relacionadas a enchentes), engenharia de } \\
\text { saúde pública, assistência farmacêutica (enviados } 1.330 \text { kits de medicamentos } \\
\text { e insumos estratégicos), assessoria técnica às secretarias de saúde e orienta- } \\
\text { ção sobre cuidados com a saúde em situações de enchentes. }\end{array}$ \\
\hline 2008 & $\begin{array}{l}\text { Inundações e } \\
\text { deslizamentos }\end{array}$ & $\begin{array}{l}\text { Chuvas intensas combinada com ventos } \\
\text { provocaram inundações e deslizamentos } \\
\text { em Santa Catarina, com maior impacto no } \\
\text { Vale do Itajaí. No estado, } 48 \text { municípios } \\
\text { foram atingidos e } 14 \text { decretaram estado de } \\
\text { calamidade pública. Somaram-se mais de } \\
88 \text { mil pessoas atingidas (desabrigados/ } \\
\text { desalojados) e } 135 \text { óbitos. Os danos mate- } \\
\text { riais foram diversos. } \\
\text { Foram registrados danos em unidades de } \\
\text { saúde. }\end{array}$ & $\begin{array}{l}\text { Mobilizado o COE para o monitoramento do evento e disponibilização de } \\
\text { equipe técnica para apoiar as ações das secretarias de saúde, recursos } \\
\text { financeiros; assessoramento técnico; envio de laboratório móvel para } \\
\text { análise de água, } 180 \text { kits de medicamentos e insumos estratégicos; } 100 \\
\text { mil fracos de hipoclorito de sódio 2,5\%; imunobiológicos e material de } \\
\text { orientação e comunicação. }\end{array}$ \\
\hline
\end{tabular}


Quadro 1. (cont.)

\begin{tabular}{lll}
\hline Ano & Evento & Principais Impactos \\
\hline 2010 & Inundações em & Fortes chuvas que atingiram a região \\
& Minas Gerais e & Sudeste deixando os estados do Rio de \\
& Espírito Santo & Janeiro, Espírito Santo e Minas Gerais com \\
& & diversos municípios atingidos.
\end{tabular}

\section{Principais Medidas}

Foi acionado o COE para o monitoramento das ações de saúde e realizadas visitas técnicas às secretarias de saúde das áreas atingidas, o envio de kits de medicamentos e insumos estratégicos e equipes de pronta-resposta.

Foi ampliado o apoio aos três estados da Região Sudeste para a intensificação das ações de vigilância epidemiológica, sanitária e ambiental, assistência farmacêutica, engenharia de saúde pública, assistência pré-hospitalar, hospitalar e ambulatorial além de assistência humanitária e atenção psicossocial às comunidades atingidas.

2011 Enxurradas e deslizamentos na Região Serrana do Rio de Janeiro

As chuvas que atingiram a Região Serrana do Rio de Janeiro, em 12 de janeiro, culminaram em um desastre de grande magnitude no Brasil, que deixou sete municípios em situação de calamidade pública, 889 óbitos, 13.741 desabrigados, 22.496 desalojados.

2012 Inundações no Acre

Chuvas intensas no início de 2012 provocaram inundações que atingiram diversos municípios no Acre de forma mais intensa em Assis Brasil, Brasiléia, Epitaciolândia, Porto Acre, Rio Branco, Santa Rosa do Purus, Sena Madureira e Xapuri.

Nesse ano, foi registrada a maior cheia no Rio Negro (29,78m); a inundação atingiu 90\% dos municípios do Amazonas, e 56 destes decretaram situação de emergência entre janeiro e junho.

Fortes chuvas atingiram o município de Lajedinho, na Bahia, que decretou estado de calamidade pública por enxurradas que provocaram a destruição de mais de 200 casas e 16 óbitos.
Mobilizado o COE com a participação de diversas áreas do MS e deslocada equipe de pronta-resposta para apoio às Secretarias de Saúde na organização da resposta ao evento. Foram mobilizadas, além de profissionais do MS e da Rede Hospitalar Federal no Rio de Janeiro também voluntários de saúde de diversos estados e da Opas/Organização Mundial da Saúde (OMS). Foram mobilizados recursos para ações de assistência à saúde, de vigilância em saúde além de orientação voltada para os profissionais que atuaram na resposta ao evento e à população atingida. Foram deslocados 8 profissionais do MS para ações de coordenação de atividades de campo; 25 profissionais do Samu Nacional; 54 profissionais de saúde da Rede Hospitalar Federal no Rio de Janeiro em Nova Friburgo; 4 profissionais de saúde da Secretaria de Saúde do Estado de São Paulo (Grau Resgate) além de equipe voluntária de apoio com 15 missionários e 1 padre).

Foi instalado um COE para o monitoramento das inundações e atender as demandas da área atingida. O MS enviou 41 profissionais para apoiar as ações de saúde e de comunicação nos municípios atingidos (Médicos: 13 - Enfermeiros: 14 - Técnicos de enfermagem: 10 - Jornalistas: 2 - Vigilância em Saúde: 2). Além disso, foram desenvolvidas atividades em abrigos, aldeias indígenas e comunidades ribeirinhas nos municípios de Santa Rosa, Porto Acre, Brasileia, Xapuri, Sena Madureira, Assis Brasil e Rio Branco. As equipes assistenciais da Força Nacional do SUS (FN-SUS) realizaram em torno de 1.400 atendimentos médicos. A missão ficou em campo por 21 dias e foi desmobilizada no dia 10/3/2013.

O MS passou a monitorar o estado do Amazonas também no COE e enviou equipes em missão exploratória ao estado para apoiar na análise de situação de saúde dos municípios atingidos e apoiar as secretarias de saúde na resposta à emergência, com destaque para ações de avaliação da rede de atenção à saúde (básica e hospitalar), intensificação da vigilância em saúde especialmente em malária, DDA, leptospirose, dermatites, febre tifoide e acidente com animais peçonhentos. Foram disponibilizados recursos financeiros para a instalação de balsa-hospitais, enviados 47 kits de medicamentos e insumos estratégicos, antecipada cota de hipoclorito de sódio 2,5\% e reposição de soro antiofídico.

O MS enviou um profissional da FN-SUS para apoiar a avaliação de danos e verificar as necessidades na área da saúde para atendimento dos afetados. A partir disso, foi implantado um posto de saúde temporário em uma escola do município, enviada equipe composta por: 2 médicos, 2 enfermeiros, 2 técnicos de enfermagem, 2 condutores de veículos de emergência, 0 ambulâncias $4 \times 4$ e 1 kit de medicamentos e insumos estratégicos com capacidade de atendimento de 1.500 pessoas.

A equipe da FN-SUS atuou em conjunto com as equipes de PSF do município e dos municípios vizinhos, realizando atendimento da demanda espontânea no Posto Médico provisório. Além disso, foram realizadas visitas domiciliares com fornecimento de novas receitas aos doentes crônicos, liberação de medicamentos de uso contínuo, distribuição e orientação quanto ao uso de hipoclorito de sódio 2,5\% e, cuidados com a saúde para reduzir o risco de doenças diarreicas e leptospirose. 
Quadro 1. (cont.)

\begin{tabular}{|c|c|c|c|}
\hline Ano & Evento & Principais Impactos & Principais Medidas \\
\hline 2014 & $\begin{array}{l}\text { Inundações na } \\
\text { Região Norte - } \\
\text { Rondônia e Acre }\end{array}$ & $\begin{array}{l}\text { As inundaç̃oes no Rio Madeira atingiram } \\
\text { os estados de Rondônia, Pará, Acre e Ama- } \\
\text { zonas, provocaram danos e interditaram } \\
\text { o tráfego na BR } 364 \text {, isolando o acesso } \\
\text { terrestre ao Acre. Em Rondônia, os municí- } \\
\text { pios mais atingidos foram Guajará-Mirim e } \\
\text { Nova Mamoré. }\end{array}$ & $\begin{array}{l}\text { O MS mobilizou o COE para o monitoramento das ações de saúde nos es- } \\
\text { tados de Rondônia e Acre, mais atingidos. Dentre as ações desenvolvidas, } \\
\text { destacam-se: } \\
\text { - Missão Exploratória em ação conjunta com a Defesa Civil para avaliação de } \\
\text { danos; } \\
\text { - Apoio à instituição da sala de crise da saúde com reuniões diárias; } \\
\text { - Identificação de impacto à rede de atenção básica por prejuízo na capaci- } \\
\text { dade de deslocamento dos profissionais de saúde até as unidades de atendi- } \\
\text { mento. } \\
\text { - Envio de } 10 \text { profissionais da FN-SUS (médicos, enfermeiros e técnicos de } \\
\text { enfermagem) para ações assistenciais, de promoção e prevenção, diagnósti- } \\
\text { cos e tratamento, além de apoio à gestão para organização das unidades de } \\
\text { saúde; } \\
\text { - Envio de } 25 \text { kits de medicamentos e insumos estratégicos; } \\
\text { - Apoio às ações de assistência e vigilância aos atingidos nos municípios de } \\
\text { Guajará-Mirim e Nova Mamoré/RO, incluindo ações de saúde em abrigos. }\end{array}$ \\
\hline 2017 & $\begin{array}{l}\text { Inundações e } \\
\text { Enxurradas nos } \\
\text { estados do Ama- } \\
\text { zonas, de Alagoas, } \\
\text { de Pernambuco, do } \\
\text { Paraná, de Santa } \\
\text { Catarina e do Rio } \\
\text { Grande do Sul }\end{array}$ & $\begin{array}{l}\text { Instituído COE para o monitoramento de } \\
\text { inundações e enxurradas que atingiram } \\
229 \text { municípios nos seis estados, sendo o } \\
\text { Rio Grande do Sul o mais afetado com } 131 \\
\text { municípios em situação de emergência } \\
\text { seguido do Amazonas (39), de Alagoas e } \\
\text { de Pernambuco ( } 27, \text { cada), do Paraná (3) e } \\
\text { de Santa Catarina (2). } \\
\text { O evento deixou desabrigados e desalo- } \\
\text { jados em diversos municípios e culminou } \\
\text { em } 18 \text { óbitos, sendo que, destes, } 8 \text { foram } \\
\text { registrados em Alagoas, } 5 \text { em Pernambuco } \\
\text { e } 5 \text { no Rio Grande do Sul. } \\
\text { A situação mais crítica foi identificada em } \\
\text { Alagoas e em Pernambuco. }\end{array}$ & $\begin{array}{l}\text { Foram deslocadas equipes técnicas para Alagoas e Pernambuco para } \\
\text { apoio às accões desenvolvidas pelas Secretarias de Saúde. Além disso, } \\
\text { foram enviados: } \\
\text { - } 111 \text { kits de medicamentos e insumos estratégicos: Alagoas (27), Amazo- } \\
\text { nas (45), Pernambuco (28) e Rio Grande do Sul (11); } \\
\text { - Unidades Móveis para o Controle da Qualidade da Água (UMCQA) da } \\
\text { Funasa: Alagoas e Pernambuco; } \\
\text { - Missão exploratória de técnicos da Força Nacional do SUS, tendo em } \\
\text { vista apoiar a organização das ações de resposta no Estado; } \\
\text { - Envio de insumos laboratoriais, doses de dT, soros antibotrópicos, soros } \\
\text { anticrotálicos, vacinas antirrábica humana e para Hepatite B. } \\
\text { - Instalação de Hospital de Campanha das forças armadas em Alagoas. }\end{array}$ \\
\hline
\end{tabular}

Fonte: Elaboração própria.

As inundações são eventos recorrentes no Brasil e no mundo, causam impactos sobre a saúde das pessoas, podem comprometer a capacidade de atendimento; e a preparação prévia possibilita uma atuação oportuna, reduzindo os riscos à saúde.

Apesar de ser consenso no discurso governamental e da comunidade científica de que a saúde é atingida pelas inundações de forma direta e indireta e em diferentes temporalidades, não são apresentadas evidências epidemiológicas consistentes de causa-efeito, e dados não registrados e subestimados são apontados como o problema de não se encontrar essa relação $\mathbf{7}^{\mathbf{1 9}, 22}$.

A distribuição espaço-temporal das inundações no Brasil demonstra que todas as regiões são atingidas e que é necessário implementar a estratégia de preparação e resposta à ESP por desastres para que se amplie a capacidade de atuação nas três esferas de gestão do SUS ${ }^{22,26}$, principalmente a local, o que é apontado também em estudos que analisaram impactos sobre a saúde 12,26,27. Eventos que superem a capacidade instalada nas Secretarias de Estado da Saúde (SES) podem acionar apoio adicional do MS, no entanto, considerando a dimensão continental do País, é imprescindível o investimento em iniciativas de gestão do risco de desastres e que esta seja transversal, com foco na instância municipal. Essa é uma das principais recomendações presentes nos marcos internacionais ${ }^{18}$ e nacionais ${ }^{11,14}$ e evidenciada por pesquisadores ${ }^{\mathbf{2}, 4,19,22}$. 
A busca pela redução do risco de desastres tem sido amplamente discutida pelos países signatários das Nações Unidas, e isso se ampliou a partir do estabelecimento da década de 1990 como a Década Internacional de Redução de Desastres, quando os países foram chamados a definir normativas e a desenvolver ações para que se reduzisse a ocorrência desses eventos e, principalmente, dos danos deles decorrentes ${ }^{\mathbf{1 8}, \mathbf{1 9}}$. O Brasil foi um dos países que buscaram estabelecer políticas públicas para a gestão de risco de desastres em diversos setores, incluindo o da saúde, harmonizando-as com o preconizado também pelo Regulamento Sanitário Internacional (RSI) quando este define evento como uma manifestação de doença ou ocorrência com potencial para causar doença; neste caso, estão as inundações ${ }^{\mathbf{1 4}, 19}$.

Ao longo desse processo, a ocorrência de eventos de grande impacto induziu a adoção de medidas normativas e norteadoras da atuação na gestão de risco de desastres. Após a resposta às inundações que atingiram todo o País em 2004-2005, foi estabelecido o kit de medicamentos e insumos estratégicos para atendimento aos municípios atingidos por desastres, Portarias no 405 e $\mathrm{n}^{\circ} 2.132$, e instituída a Comissão de Desastres, Portaria ${ }^{0}$ 372, todas do gabinete do Ministro da Saúde publicadas em 2005, hoje regulamentados pela Portaria de Consolidação $n^{0} 1 / 2017$, Capítulos IV, seções I e II.

Após as fortes chuvas que atingiram a região Sul em 2008, com impactos significativos em Santa Catarina ${ }^{28}$, a discussão da necessidade de ampliar a capacidade de atuação do SUS em desastres teve mais visibilidade, dessa forma, foram elaborados materiais norteadores para as secretarias de saúde e ampliados os processos de capacitação.

Em 2010, entre as ações inseridas na Programação de Vigilância em Saúde (Pavs) - e com vistas ao fortalecimento da atuação das SES em desastres -, foi recomendada pelo MS a instituição de Comitês Estaduais de Saúde em Desastres (Cesd), de forma a organizar a preparação e resposta das SES, considerando as respectivas especificidades locais ${ }^{5,11}$.

Após o desastre da Região Serrana do estado do Rio de Janeiro, em 2011, criaram-se a Força Nacional do SUS (FN-SUS) e a Declaração de Emergência em Saúde Pública de Importância Nacional, por meio do Decreto $n^{0} 7.616 / 2011$, regulamentadas no capítulo III da mesma Portaria. Em 2014, foram publicados o Plano de Resposta à Emergência em Saúde Pública e, além de outros, o Plano de Contingência para resposta a Inundações seguidas de diversas outras iniciativas no SUS voltadas para saúde em desastres.

Quando comparadas as ações desenvolvidas na resposta às inundações de 2004 e as demais até 2017 , observa-se que foram estabelecidos mecanismos normativos e documentais para melhorar a atuação do MS no atendimento às secretarias de saúde atingidas por esses eventos, incluindo ferramentas de monitoramento, análise da situação e gestão das emergências.

Analisando os desafios e avanços alcançados ao longo da história, fica evidente que há uma mudança de paradigma em que pode ser observado que as ações hoje desenvolvidas não se restringem à atuação pós-desastres e envolvem iniciativas para a redução do risco e a preparação para resposta à emergência, tendo ampliado o fortalecimento da capacidade de atuação em nível local. O Setor Saúde hoje tem o seu papel reconhecido, tanto no Sistema Nacional de Proteção e Defesa Civil quanto no próprio SUS, como um protagonista na gestão de risco de desastres; dessa maneira, a articulação com outros atores dessa agenda tem sido ampliada, possibilitando uma atuação interinstitucional e multidisciplinar.

Os eventos de grande magnitude exigem rápida mobilização de esforços para a resposta à emergência e possibilitam identificar os pontos críticos a serem adequados. A experiência advinda desse cenário pode resultar em alguns dos aspectos que influenciam a adoção de medidas subsequentes a essas ocorrências, a exemplo da regulamentação legal, do 
estabelecimento de políticas, estratégias e programas de gestão do risco de desastres em âmbito federal, estadual e municipal.

É inegável que a gestão de risco de desastres no Brasil tem-se ampliado, no entanto, apresenta-se como necessária a institucionalização das articulações intersetoriais e interinstitucionais, que, muitas vezes, são focadas em relações interpessoais. Garantir a formalização dessas iniciativas permite a continuidade do trabalho mesmo que haja alterações dos profissionais envolvidos na agenda.

Nesse contexto, torna-se um desafio dotar o SUS da capacidade necessária para o monitoramento, a detecção e o controle de eventos que se apresentem com potencial de risco à saúde, possibilitando o desenvolvimento de um conjunto de ações, de forma contínua, que inclua o planejamento e a adoção de medidas de prevenção, preparação, resposta e recuperação, envolvendo os diversos segmentos do setor de saúde e de outros setores envolvidos nessa agenda. A descontinuidade ou intermitência do processo de gestão do risco de desastres dificulta a implementação das medidas necessárias que possibilitam uma atuação oportuna, principalmente no nível local, que é onde os eventos se materializam e onde ocorre a primeira resposta.

\section{Colaboradores}

Silva EL (0000-0003-4608-5946)*, Resende RMS (0000-0002-0813-3751)*, Frutuoso RL (0000-0001-7270-8807)*, Bezerra AB (00000001-9491-0336)*, Salvi BB (0000-0002-24019190)*, Rohlfs DB (0000-0001-6967-0852)* contribuíram igualmente para a elaboração do manuscrito.

\section{Referências}

1. Organização Pan-Americana de Saúde. Desastres Naturais e Saúde no Brasil [internet]. 2. ed. Brasília, DF; 2015. [acesso em 2019 abr 6]. Disponível em: https://www.paho.org/bra/images/stories/GCC/ desastresesaudebrasil_2edicao.pdf.

2. Freitas CM, Silva DRX, Sena ARM, et al. Desastres naturais e saúde: uma análise da situação do Brasil. Ciênc. Saúde Colet. 2014 [acesso em 2020 jan 20]; 19(9):3645-3656. Disponível em: http://www. scielo.br/scielo.php?script=sci_arttext\&pid=S1413$-81232014000903645 \& \operatorname{lng}=\mathrm{en}$.
3. Organização Pan-Americana da Saúde. Los Desastres Naturales y Proteccion de La Salud. Publicació [internet]. Washington, D.C.: OPS; 2000 [acesso em 2019 abr 15]. Disponível em: http://iris.paho.org/xmlui/ handle/123456789/748.

4. Noji EK. Impacto de Los Desastres En La Salud Pública. Bogotá: OPAS; 2000.

5. Brasil. Ministério da Saúde. Plano de Contingência para Emergência em Saúde Pública por Inundação. 2014 [acesso em 2019 abr 12]. Disponível em: http://
*Orcid (Open Researcher and Contributor ID). 
bvsms.saude.gov.br/bvs/publicacoes/plano_contingencia_emergencia_saude_inundacao.pdf.

6. Castro ALC. Glossário de Defesa Civil: Estudos de Riscos e Medicina de Desastres [internet]. 5. ed. Brasília, DF: Ministério da Integração Nacional/ Secretaria Nacional de Defesa Civil; 2002. Disponível em: http://www.rladr.mdr.gov.br/c/document_library/get_file?uuid=71458606-5f48-462e8f03-4f61de3cd55f\&groupId=10157.

7. Ahern M, Kovats RS, Wilkinson P, et al. Global health impacts of floods: Epidemiologic evidence. Epidemiol Rev. 2005 [acesso em 2020 jan 20]; (27):36-46. Disponivel em: https://www.ncbi.nlm.nih.gov/pubmed/15958425.

8. Ceped. Atlas Brasileiro de Desastres Naturais: 1991 a 2012 [internet]. [acesso em 2019 jan 22]. Disponível em: https://www.ceped.ufsc.br/wp-content/uploads/2012/01/AMAZONAS_mioloWEB.pdf.

9. Brasil. Ministério da Saúde. Desastres naturais e saúde: análise do cenário de eventos hidrológicos no Brasil e seus potenciais impactos sobre o Sistema Único de Saúde [internet]. Bol Epidemiológico da Secr Vigilância em Saúde. 2018 [acesso em 2019 abr 9]; 49:13. Disponível em: http://portalarquivos2.saude. gov.br/images/pdf/2018/marco/22/2017-032-Publicacao.pdf.

10. Freitas CM, Ximenes EF. Enchentes e saúde pública: uma questão na literatura científica recente das causas, consequências e respostas para prevenção e mitigação. Ciênc. Saúde Colet. 2012 [acesso em 2020 jan 20]; 17(6):1601-1616. Disponível em: http://www. scielo.br/scielo.php?script=sci_arttext\&pid=S1413$-81232012000600023 \& \operatorname{lng}=\mathrm{en}$.

11. Brasil. Ministério da Saúde. Guia de Preparação e Resposta à Emergência em Saúde Pública por Inundação [internet]. 2017 [acesso em 2019 abr 6]. Disponível em: http://bvsms.saude.gov.br/bvs/publicacoes/ guia_preparacao_respostas_emergencia_saude_publica_inundacao.pdf.
12. Londe LR, Coutinho MP, Di Gregório LT, et al. Desastres relacionados à água no Brasil: perspectivas e recomendações. Ambient Soc. 2015; 17(4):133-152.

13. Fatemi F, Ardalan A, Aguirre B, et al. Social vulnerability indicators in disasters: Findings from a systematic review. Int J Disaster Risk Reduct. 2017; 22:219227.

14. Brasil. Ministério da Saúde. Plano de Resposta às Emergências em Saúde Pública [internet]. 2014 [acesso em 2019 abr 10]. Disponível em: http://bvsms.saude.gov.br/bvs/publicacoes/plano_resposta_emergencias_saude_publica.pdf.

15. Caruso GD. The legacy of natural disasters: The intergenerational impact of 100 years of disasters in Latin America. J Dev Econ. 2017; 127:209-233.

16. Centre for Research on the Epidemiology of Disasters; United Nations Office for Disaster Risk Reduction. Economic Losses, Poverty and Disasters 19982017. 2018. [acesso em 2020 maio 14]. Disponível em: https://www.preventionweb.net/files/61119_credeconomiclosses.pdf.

17. Freitas CMD, Silva DRX Sena ARMD. Desastres naturais e saúde: uma análise da situação do Brasil. Ciênc. Saúde Colet. 2014; (9):3645-3656.

18. UNISDR. Marco de Sendai para la Reducción del Riesgo de Desastres 2015-2030. Organização das Nações Unidas. 2015 [acesso em 2019 abr 6]. Disponível em: https://www.unisdr.org/files/43291_spanishsendaiframeworkfordisasterri.pdf.

19. Ugarte C, Aguilar P, Mauvernay J. Voluntad política, coordinación y planificación: componentes clave para fortalecer la respuesta nacional a emergencias y desastres de salud pública en países de América Latina y el caribe. Rev Panam Salud Pública. 2018; (42):1-4.

20. Few R, Ahern M, Matthies F, et al. Floods, Health and Climate Change: A Strategic Review. 2004. [acesso em 2019 dez 20]. Disponível em: https://www.unisdr.org/files/1985_VL206506.pdf. 
21. Bich TH, Quang LN, Ha LTT, et al. Impacts of flood on health: epidemiologic evidence from Hanoi, Vietnam. Glob Health Action. 2011 [acesso em 2019 dez 20]; 4(1):6356. Disponível em: https://www.tandfonline.com/doi/full/10.3402/gha.v4i0.6356.

22. Freitas CM, Carvalho ML, Ximenes EF, et al. Vulnerabilidade socioambiental, redução de riscos de desastres e construção da resiliência - lições do terremoto no Haiti e das chuvas fortes na Região Serrana, Brasil. Ciênc. Saúde Colet. 2012; 17(6):1577-1586.

23. Brasil. Lei ${ }^{\circ} 12.608$, de 10 de abril de 2012. Brasil; 2012. Institui a Política Nacional de Proteção e Defesa Civil - PNPDEC; dispõe sobre o Sistema Nacional de Proteção e Defesa Civil - SINPDEC e o Conselho Nacional de Proteção e Defesa Civil - CONPDEC; autoriza a criação de sistema de informações e monitoramento de desastres; altera as Leis $\mathrm{n}^{\circ} 12.340$, de $1^{\circ}$ de dezembro de 2010, 10.257, de 10 de julho de 2001, 6.766, de 19 de dezembro de 1979, 8.239, de 4 de outubro de 1991, e 9.394, de 20 de dezembro de 1996; e dá outras providências. Diário Oficial da República Federativa do Brasil. 2012 Abr 11. [acesso em 2019 nov 9]. Disponível em: http://www.planalto. gov.br/ccivil_03/_Ato2011-2014/2012/Lei/L12608. htm\#: :text=Institui\%20a\%20Pol\%C3\%ADtica\%20 Nacional\%20de,1996\%3B\%20e\%20d\%C3\%A1\%20 outras\%20provid\%C3\%AAncias.
24. Brasil. Plano Nacional de Gestão de Riscos e Resposta a Desastres. Brasília, DF; 2012. [acesso em 2019 dez 20]. Disponível em: http://www.planejamento.gov. br/apresentacoes/apresentacoes-2012/120808_plano_nac_risco_2.pdf.

25. Brasil. Ministério da Saúde. Relatório das ações de resposta às enchentes de 2004. Brasília, DF: Ministério da Saúde; 2004.

26. Minervino AC, Duarte EC. Danos materiais causados à Saúde Pública e à sociedade decorrentes de inundações e enxurradas no Brasil, 2010-2014: dados originados dos sistemas de informação global e nacional. Ciênc. Saúde Colet. 2016; 21(3):685-694.

27. Freire NCF, Bonfim CV, Natenzon CE. Vulnerabilidade socioambiental, inundações e repercussões na Saúde em regiões periféricas: o caso de Alagoas, Brasil. Ciênc. Saúde Colet. 2014; 19(9):3755-3762.

28. Xavier DR, Barcellos C, Freitas CM. Eventos climáticos extremos e consequências sobre a saúde: o desastre de 2008 em Santa Catarina segundo diferentes fontes de informação. Ambient Soc. 2015; 17(4):273294.

Recebido em 16/08/2019

Aprovado em 18/12/2019

Conflito de interesses: inexistente

Suporte financeiro: não houve 